\title{
Jung, Beneduce e i primi anni dell'Iri (1932-1936)
}

\author{
Nicola De Ianni*
}

\section{Jung: da nazionalista a mussoliniano}

Guido Jung nacque a Palermo nel 1876. Era un nazionalista convinto ${ }^{1}$. La sua origine triestina, da parte di madre, ne fece un irredentista e dall'estate del 1914 un interventista, schierato con D'Annunzio, contro il giolittismo e la Triplice intesa.

Tra i primi decise di andare volontario in guerra. Vi resterà per oltre tre anni e mezzo, guadagnando tre medaglie d'argento al valore, l'ultima, dopo Caporetto, quando fu tra coloro che eseguirono l'ordine di sparare alla schiena ai soldati che fuggivano ${ }^{2}$.

* Università degli studi di Napoli Federico II. Alla figura di Guido Jung, prima della voce sul Dizionario biografico degli italiani (vol. LXII, Istituto della Enciclopedia italiana, Roma 2004), di alcuni interventi successivi e della biografia Il ministro soldato del 2009, non era stata accordata una considerazione adeguata allo spessore del personaggio. Si sapeva, per merito di Lucio Villari, dell'esistenza di un fondo assai ricco sulla ditta Jung e su Guido Jung, messo in ordine poco prima della sua morte da Guido e da suo nipote Mario. Il compianto Ugo Jung, figlio di Mario, assecondando le mie sollecitazioni, accettò di mettere a disposizione degli studiosi il fondo, donandolo all'Archivio storico della Banca d'Italia. Le fonti documentarie per la ricostruzione dell'attività di Alberto Beneduce sono numerose e ricchissime. Punto obbligato di partenza è il Fondo Alberto Beneduce presso l'Archivio storico della Banca d'Italia.

1 N. De Ianni, Il ministro soldato. Vita di Guido Jung, Rubbettino, Soveria Mannelli 2009, pp. 49-59.

2 Ivi, pp. 90-91.

Nicola De lanni, University of Naples Federico II, Italy, deiannin@tin.it FUP Best Practice in Scholarly Publishing (DOI 10.36253/fup_best_practice)

Nicola De lanni, Jung, Beneduce e i primi anni dell'Iri (1932-1936), pp. 117-140, @ 2020 Author(s), CC BY 4.0 International, DOI 10.36253/978-88-5518-202-7.07, in Piero Barucci, Piero Bini, Lucilla Conigliello (edited by), I mille volti del regime. Opposizione e consenso nella cultura giuridica, economica e politica italiana tra le due guerre, ( 2020 Author(s), content CC BY 4.0 International, metadata CC0 1.0 Universal, published by Firenze University Press (www.fupress.com), ISSN 2704-5919 (online), ISBN 978-88-5518202-7 (PDF), DOI 10.36253/978-88-5518-202-7 
Per le buone relazioni internazionali, per la preparazione sulle questioni finanziarie e per l'ottima conoscenza della lingua inglese, rappresentò l'Italia alla Conferenza della Pace di Parigi e poi nelle successive conferenze economiche del dopoguerra.

Tra i primi atti del governo Mussolini ci fu quello di rimuovere l'ambasciatore a Washington, Vittorio Rolandi Ricci ${ }^{3}$ e sostituirlo con Gelasio Caetani, deputato nazionalista, ingegnere minerario, latifondista romano, capitano di complemento, pluridecorato. Mussolini decise di inviare Jung, ufficialmente con la carica di consulente finanziario dell'Ambasciatore e ministro plenipotenziario, in realtà con il preciso compito di affrontare la delicata questione del debito di guerra italiano con gli Stati Uniti.

La ricostruzione delle vicende del Banco di Roma è stata, in sede storiografica, ampiamente trattata (Lugi De Rosa, Toniolo, Falco) ${ }^{4}$, non altrettanto il ruolo di Jung nella vicenda, sia a causa della possibilità di utilizzo solo recente dell'Archivio Jung, sia, in buona parte, per le omissioni di De' Stefani nel suo Baraonda bancaria ${ }^{5}$. Il ruolo di Jung risulta, invece, chiaramente in una nota autobiografica redatta in terza persona in cui si legge che nell'agosto del 1923 egli ebbe direttamente dal Capo del governo l'incarico di sistemare la situazione del Banco di Roma «da tempo profondamente travagliata $»^{6}$. A Jung è attribuibile la scelta di Sinigaglia alla presidenza della società finanziaria di smobilizzo delle partecipazioni e il delicato ruolo di mediatore tra Stringher ${ }^{7}$ e De' Stefani.

Nel gennaio 1924 Jung riprese i contatti con Alberto Pirelli per la commissione incaricata della redazione del Piano Dawes sulle riparazioni ${ }^{8}$. Anche qui egli svolse un ruolo di mediazione fra le parti con Pirelli (Confindustria), Stringher (Banca d'Italia) e De' Stefani (Ministro delle finanze). Il lavoro degli esperti

3 Mussolini aveva pensato ad Albertini, come scrisse a Pirelli. A. Pirelli, Taccuini, 1922/1943, a cura di D. Barbone, il Mulino, Bologna 1984, p. 50. Su Rolandi Ricci si veda la voce sul Dizionario biografico degli italiani di G. Grimaldi, vol. LXXXVIII, Istituto della Enciclopedia italiana, Roma 2017.

4 L. De Rosa, Storia del Banco di Roma, vol. II (dal 1911 al 1928), Banco di Roma, Roma 1982, pp. 30-39; G. Falco, Crisi bancarie e trasformazioni del sistema finanziario italiano tra prima guerra mondiale e restaurazione monetaria. Il Banco di Roma, in G. Procacci et al., Assistenzialismo e politiche di controllo sociale nell'Italia liberale e fascista, Università degli studi di Modena e Reggio Emilia, Modena 2001, pp. 151-177; G. Toniolo, Il profilo economico, in G. Guarino e G. Toniolo (a cura di), La Banca d'Italia e il sistema bancario, 1919-1936, Laterza, Roma-Bari 1993, pp. 30-39.

5 A. De' Stefani, Baraonda bancaria, Edizioni del Borghese, Milano 1960. Non sorprendono le omissioni di De' Stefani, considerata la sua tendenza accentratrice.

6 Archivio Jung, Palermo, corda 9, fasc. 12, f. 21.

7 Sull'autorevolezza di Stringher e sulla sua capacità di stabilire con la comunità finanziaria, da cui era riconosciuto come guida, solidi rapporti fiduciari basati su regole scritte e non, si veda F. Bonelli, Introduzione, in Id. (a cura di), La Banca d'Italia dal 1894 al 1913. Momenti della formazione di una banca centrale, Laterza, Roma-Bari 1991, pp. 3-114.

8 Archivio Alberto Pirelli, Pirelli a Jung, 15/1/1924, Copia completissima dei documenti raccolti dal Segretariato generale della Commissione per le riparazioni. Ringrazio Donato Barbone per avermi fornito una copia del documento. 
della commissione si concluse con la convocazione della conferenza di Londra nell'agosto del 1924.

Jung si occupò anche di petrolio, con Caetani, dal 1923 cioè sin da quando era a Washington. L'ambasciatore sponsorizzò l'offerta della società americana Sinclair di assumere la concessione della ricerca petrolifera sul suolo italiano ${ }^{9}$ e al suo rientro in Italia fu incaricato delle trattative che porteranno alla firma della convenzione nell'aprile 1924. Jung e Caetani, partendo dal presupposto che l'Italia non aveva intenzione di investire nella ricerca, stimarono l'operazione conveniente. La convenzione Sinclair fu collegata al caso Matteotti e finì per suscitare accese polemiche che portarono Mussolini ad annullarla nel gennaio del $1925^{10}$. Dalla primavera del 1926 si passò all'opzione statalista con la nascita dell'Agip (Agenzia generale industria petroli). Occorre aggiungere che né Jung, né Caetani furono mai toccati dallo scandalo delle tangenti, né è plausibile pensare che potessero far da scudo a quelle incassate da altri uomini politici.

Chiariamo subito che gli incarichi di Jung non lo posero direttamente a contatto con il problema della lira, perlomeno fino a quando De' Stefani fu al ministero e cioè fino all'estate del 1925. Mussolini si deciderà al cambio della guardia, sostituendo De' Stefani con Volpi, anche a causa della crisi della lira, che il 3 luglio arriverà a 138 contro la sterlina.

Da quel momento si interruppero i rapporti di Mussolini con De' Stefani, mentre migliorarono ulteriormente quelli con Jung e con Stringher. Questi, nel 1928, sarà il primo governatore della Banca d'Italia, dopo l'istituzione della carica. Jung rimarrà tra i più ascoltati consiglieri del duce anche con Volpi, partecipò al progetto istitutivo dell'Ine e dal 1927 ne diventerà il presidente ${ }^{11}$.

Ma torniamo al 1925. Tra l'ottobre e il dicembre si svolse a Washington il negoziato con gli Stati Uniti per il consolidamento dei debiti di guerra. L'intesa raggiunta ricalcava quella di Caetani e Jung del 1923. Fermo il principio sul riconoscimento del debito, gli americani concedevano una lunga diluizione e una progressività crescente delle annualità compatibile con la crisi economica del Paese. Nel gennaio 1926 si perfezionò anche l'accordo con la Gran Bretagna, sulle stesse basi di quelle con il governo americano.

Per l'Italia la sistemazione dei debiti di guerra era finalizzata alla battaglia per la difesa della lira cominciata col discorso di Mussolini, a Pesaro, il 18 agosto 1926 . Prima di allora, a metà maggio del 1926, il cambio della lira riprese progressivamente a peggiorare, fino a raggiungere, tra fine luglio e fine agosto, il livello di 150, dopo il quale ci fu l'intervento di Mussolini e Volpi. Messe al sicuro le riserve di liquidità, grazie al prestito Morgan di cento milioni di dollari, si avviò una progressiva stretta monetaria che ridusse la circolazione, rese

9 M. Pizzigallo, Alle origini della politica petrolifera italiana. 1920-1925, Giuffrè, Milano 1981; Id., L'Agip degli anni ruggenti (1926-1932), Giuffrè, Milano 1984; Id., La politica estera dell'Agip, 1933-1940. Diplomazia economica e petrolio, Giuffrè, Milano 1992.

10 Archivio Jung, Palermo, corda 10, fasc. 4, ff. 1-62, Relazione per l'approvazione della convenzione Sinclair in Parlamento, giugno 1924.

11 De Ianni, Il ministro soldato, cit., p. 240. 
il denaro più caro e avviò l'inversione di tendenza. Pochi immaginavano che si sarebbe arrivati, alla fine del 1927, al momento del ritorno all'oro, a quota 92 e cioè con una rivalutazione di circa il $40 \%$ dai minimi.

Il contributo di Jung all'elaborazione della politica di 'quota novanta' s'inquadra nello schema già tracciato di crescente consolidamento del rapporto con Mussolini. Questi lo raccomandò a Volpi e gli affidò, nel luglio del 1926, uno studio «circa le ripercussioni di una stabilizzazione sull'industria, sul commercio e sulle banche», i cui risultati furono superati dagli eventi che portarono alle dichiarazioni di Pesaro. Jung aveva, però, fatto in tempo a raccomandare tanto a Mussolini, quanto a Volpi, di intervenire sulla circolazione. Le linee guida del suo studio, così come quelle di una conferenza tenuta a Palermo nel marzo del 1928 , coincidono perfettamente con quelle dell'azione di governo ed indicano che esisteva un gruppo coeso che spingeva per la rivalutazione. Di esso Mussolini si servì per lanciarsi nelle sue proverbiali intuizioni economiche ${ }^{12}$.

L'Istituto nazionale per l'esportazione (Ine) nacque per iniziativa di Pirelli e Jung, nell'estate del 1926, con il compito di promuovere l'esportazione italiana all'estero nel difficile momento di avvio della politica di rivalutazione della moneta. L'Istituto avrebbe potuto dare indicazioni circa la misura della rivalutazione, ma in sostanza cercherà soltanto di migliorare l'organizzazione ed i servizi a disposizione degli esportatori per far fronte al peggioramento delle condizioni di cambio.

Pirelli ne fu il presidente per il primo anno, per lasciare poi, dopo la nomina a presidente della Associazione delle Camere di commercio internazionali, nel luglio del 1927, il suo posto a Jung. Il cambio della guardia all'Ine registrò un oggettivo avanzamento di Jung nelle gerarchie di potere soprattutto per il sacrifico, anche personale e la disciplina di soldato, mostrati nel seguire una linea che danneggiava la sua ditta esportatrice di prodotti siciliani (mandorle, nocciole, pistacchi ecc.).

La crisi borsistica di Wall Street dell'ottobre del 1929 si trasferì nell'economia statunitense e poi, presto, nel resto del mondo. La percezione di essa come di una crisi senza precedenti per intensità ed effetti cominciò ad aversi in Italia con qualche ritardo, insieme con la speranza che colpisse meno violentemente il nostro Paese.

La designazione come rappresentante italiano della nuova Banca dei regolamenti internazionali costrinse Beneduce, nell'ottobre del 1930, a rassegnare le dimissioni dal Comitato dell'oro, costituito per il ritorno del sistema aureo nel dopoguerra, e al posto di Beneduce venne chiamato Jung.

Nel febbraio del 1931, Alberto Pirelli, in totale segretezza, si adoperò con Mussolini e probabilmente in parte con Jung, per il salvataggio del Credito ita-

12 Archivio Jung, Palermo, corda 12, fasc. 8, Stabilizzazione della moneta, conferenza tenuta a Palermo il 7 marzo 1928 presso il Circolo di studi economici. Scrive Jung: «Come sempre il genio e l'intuito infallibile dell'Uomo provvidenziale che regge destini della Patria, aveva determinato l'elemento base della situazione monetaria, e agendo sopra di esso aveva invertito la situazione della lira» (ivi, f. 17). 
liano con modalità simili a quelle che verranno adottate successivamente per la Comit. La situazione economica internazionale peggiorava e fece registrare nel maggio del 1931 la chiusura del Credit-Anstalt.

L'estate del $1931 \mathrm{fu}$ caldissima per l'economia italiana e internazionale sia per la situazione della Banca commerciale, che aprì la strada ad un intervento statale, sia per la crisi della sterlina. La prima si manifestò l'11 settembre del 1931 quando, nell'incontro romano fra Mussolini e Toeplitz, l'amministratore delegato della Comit chiese un deciso intervento per scongiurare la grave crisi di liquidità e la prossima chiusura degli sportelli $i^{13}$. La seconda si manifestò il 21 settembre del 1931 quando il governo britannico sospese la convertibilità in oro della sua valuta. La Banca d'Italia si trovò a possedere ingenti quantità di riserve in sterline, circa 18,16 milioni (pari a 1,67 miliardi di lire), che per motivi di opportunità non si era ritenuto di smobilizzare.

La crisi della sterlina colpì le banche, e in particolare la Comit, perché svalutò da un giorno all'altro l'attivo in valuta. Si ebbe poi la costituzione dell' $I m i$, cioè di una banca a medio termine che avrebbe dovuto finanziare le aziende in orbita Comit. Si attivò, infine, lo smobilizzo della Banca commerciale italiana attraverso l'acquisto delle partecipazioni industriali da parte della Sofindit.

Dopo la scelta del capo del governo di puntare su Mayer e Jung per la presidenza dei due enti, apparve subito come obbiettivo principale quello di vincere la resistenza di Toeplitz, il vero e proprio simbolo della banca mista e privata. Toeplitz, il 31 ottobre, dopo la firma della convenzione della Comit, pensò, forse troppo ottimisticamente, che con la nascita dell'Imi era stato risolto il problema dello smobilizzo. Sventò diversi colpi, compresi quelli di sottrargli Di Veroli (per promuoverlo direttore generale dell'Imi a Roma) e di trasferire a Roma la Sofindit. Sarà Jung invece a trasferirsi a Milano «per studiare - ironizza Toeplitz scrivendone a Beneduce - il contenuto del suo futuro reame $\gg^{14}$.

La nomina alla presidenza Sofindit rappresentò per Jung un'affermazione importante. La consegna ricevuta fu quella di approfondire l'esame delle partecipazioni acquisite, garantendo l'assistenza finanziaria necessaria d'intesa con l'Imi.

L'atteggiamento di Mayer e la sua posizione critica sui finanziamenti da concedere alle aziende acquisite da Sofindit cambiò le aspettative di Toeplitz. Nel novembre del 1931 e prima ancora che Jung assumesse la carica, in una lettera a Beneduce, Toeplitz sottolineava che il ruolo della finanziaria di partecipazioni dovesse risolversi nel tutelare i suoi interessi di azionista come fatto fino ad allora dalla Comit ${ }^{15}$. Mayer, invece, dichiarò, a più riprese, che intendeva finanziare soltanto iniziative sane. Jung, che pure, con Mussolini e Beneduce, era convin-

13 N. De Ianni, Guido Jung e lo Stato imprenditore, in A. Cova, G. Fumi (a cura di), L'intervento dello Stato nell'economia italiana. Continuità e cambiamenti (1922-1956), Franco Angeli, Milano 2011, pp. 89-90.

14 Archivio storico Intesa Sanpaolo, Banca commerciale italiana, Copialettere Toeplitz, 75/384-385, Toeplitz a Beneduce, 16/12/1931.

15 Ivi, 75/256, Toeplitz a Beneduce, 23/11/1931. 
to che il tempo giocasse a loro favore, poiché logorava la posizione di Toeplitz, tentò di attenuare le eccessive chiusure del presidente dell'Imi.

Con la dichiarazione d'inconvertibilità della sterlina, nel settembre del 1931 si inaugurò un periodo di attesa circa l'entità della svalutazione e sul comportamento del governo italiano e si riaccesero, in alcuni settori industriali e bancari, speranze di svalutazione. In nessun momento, però, Mussolini e i suoi collaboratori diedero l'impressione di voler abbandonare la scelta del 1927, anzi da 'quota novanta' si passerà a 'quota sessanta' per il rifiuto di voler seguire la sterlina nella sua svalutazione ${ }^{16}$. Dall'agosto del 1926, quando ebbe inizio la politica di rivalutazione, fino all'ottobre del 1936, quando finalmente ci sarà il 'riallineamento', le ragioni economiche furono subordinate a quelle politiche.

Il risultato finale per il fascismo fu quello della nazionalizzazione del sistema bancario italiano attraverso il capovolgimento dei rapporti di forza tra pubblico e privato. Con la nascita dell'Iri si diede vita ad un modello originale di intervento del fascismo nell'economia. Tale ordine rappresentò la difficile mediazione tra il corporativismo e le istituzioni prefasciste e trovò nel totalitarismo mussoliniano la sua forza espressiva ${ }^{17}$.

Nel luglio del 1932, quando fu chiamato alla guida del Ministero delle finanze, Jung divenne, di fatto, il principale esecutore di questo programma.

\section{Beneduce: da Nitti a Stringher fino alla silenziosa adesione al fascismo}

Beneduce nacque a Caserta, nel 1877, da una famiglia di umili origini. Si sposò a 21 anni con la ventenne Noemi e già nel 1901, a 24 anni, aveva tre figli (diventeranno poi 5). La laurea in matematica arrivò l'anno dopo e nel 1903 vinse un concorso per vicesegretario di seconda classe presso il Ministero agricoltura industria e commercio. L'ufficio dove Beneduce lavorava - presso la direzione generale di statistica - aveva la competenza delle statistiche nazionali (l'Istat nascerà soltanto nel 1926). Con Attolico, Giuffrida, Ruini, Serpieri ed altri fece parte di un gruppo di giovani dell'élite burocratica ministeriale il cui riferimento assoluto fu Francesco Nitti, al quale era stato presentato alcuni anni prima

17 Mussolini temeva alcuni esponenti del Partito nazionale fascista, tra cui il principale era certamente Farinacci, per l'uso ideologico e retorico che avrebbero potuto fare del corporativismo (si veda M. Di Figlia, Farinacci. Il radicalismo fascista al potere, Donzelli, Roma 2007). L'ideologo del corporativismo, Ugo Spirito, non era, invece, tenuto in alcuna considerazione. Stesso discorso vale per Beneduce. In proposito le osservazioni di Piero Barucci: «Il convegno di Ferrara è del 1932, quando il "duce" in persona tentava disperatamente di "risanare" il sistema bancario italiano ormai prossimo al collasso. Sono i mesi in cui Beneduce pensa all'Iri, durante i quali quelle spiritiane sembrano elucubrazioni inutili. Vien da pensare che Beneduce, il vero "duce" dell'economia italiana come fu chiamato, non abbia mai letto un rigo degli scritti di Spirito». P. Barucci, Presentazione, in P. Barucci, P. Bini, L. Conigliello (a cura di). Il corporativismo nell'Italia di Mussolini. Dal declino delle istituzioni liberali alla Costituzione repubblicana, Firenze University Press, Firenze 2018, p. XII. 
dal padre del suo amico Giorgio Mortara ${ }^{18}$. Nel 1910 conseguì la libera docenza ed entrò a far parte, con Maffeo Pantaleoni e Giorgio Mortara, della direzione del «Giornale degli economisti». Dal 1911, con Giuffrida capo di gabinetto del ministro Nitti (agricoltura, industria e commercio), Beneduce occupò la funzione di segretario particolare.

Fu iniziato alla massoneria dal fratello Ernesto nel 1905 e nel 1912 era già uno dei massimi esponenti del Grande Oriente d'Italia. Nel febbraio del 1917 assurgerà al massimo grado (il $33^{\circ}$ ) della gerarchia ${ }^{19}$.

Nel 1912 Beneduce guidò la sezione socialista del nuovo Partito socialista riformista di Caserta, dopo la scissione avvenuta al congresso di Reggio Emilia. Nel settembre 1912, fu cooptato, in qualità di fiduciario di Nitti, nel consiglio d'amministrazione dell'Istituto nazionale delle assicurazioni (Ina), costituito con il fine di assicurare al settore pubblico il monopolio delle assicurazioni sulla vita.

Per bilanciare il potere di Nitti, Giolitti scelse come presidente dell'Ina il direttore della Banca d'Italia Bonaldo Stringher che ebbe modo da allora di stabilire con Beneduce un rapporto di stima e di fiducia crescenti, durato fino alla sua morte nel 1930.

Ma torniamo al 1914. Beneduce era fortemente preoccupato per le incertezze del suo futuro lavorativo e se ne lamentò con Nitti. Sono gli stessi mesi in cui Jung, folgorato dalla scelta nazionalista, meditava di lasciare l'azienda di famiglia, che dirigeva, e partire volontario per il fronte. Anche Beneduce, sia pure su posizioni molto moderate, aderì al gruppo di socialisti favorevoli all'intervento, criticò Nitti che invece apprezzava la posizione neutralista di Giolitti e visse anche una breve stagione al fronte di due mesi e mezzo (dal 1 settembre al 17 novembre 1915) dopo di che fu messo in congedo, tra gli ufficiali esuberanti al servizio.

Nel 1914 aveva ottenuto la cattedra universitaria e nel 1917 diventò direttore generale e consigliere delegato dell'Ina. La necessità di non far mancare il suo apporto all'Ina dovette fargli accettare la guerra come una esperienza necessaria e limitata, del resto egli era ancora troppo vicino a Nitti per non prendere opportunamente le distanze da essa ${ }^{20}$.

Nel 1916 e 1917 Beneduce incoraggiò Nitti a tornare sulla scena politica, cosa che avvenne, infatti, il 30 ottobre del 1917 come Ministro del tesoro, nel gabinetto Orlando. In pochi mesi Nitti istituì il monopolio statale delle transazioni in valuta e costituì l'Istituto cambi con l'estero (Ince), azzerando i profitti speculativi che le grandi banche lucravano su queste operazioni. Contestualmente autorizzò l'emissione di una polizza speciale a favore dei militari per i rischi di guerra, creando così le basi per la nascita dell’Opera nazionale combattenti

18 M. Franzinelli, M. Magnani, Beneduce. II finanziere di Mussolini, Mondadori, Milano 2009, pp. 21-23.

19 Fino al 1924, Beneduce mantenne rapporti con il Grande Oriente d'Italia e poi, presumibilmente per evitare di compromettersi, li lasciò cadere. Si veda F. Conti, Storia della massoneria italiana. Dal risorgimento al fascismo, il Mulino, Bologna 2003.

20 N. De Ianni, Il viaggio breve. Beneduce dal socialismo al fascismo, «Rivista di storia finanziaria», 14, gennaio-giugno 2005 , p. 48. 
(Onc). Infine, il 15 gennaio 1918 lanciò il quinto prestito nazionale per un importo di sei miliardi di lire. Di tutte queste iniziative Beneduce sarà investito direttamente in qualità di amministratore dell'Ince, consigliere delegato dell'Onc, direttore dell'Ina, nonché interlocutore privilegiato e fiduciario del ministro.

Beneduce oscillava tra Nitti e Stringher. Nel gennaio del 1919 quando Nitti si dimise per contrasti con Sonnino ed Orlando, Stringher fu chiamato a sostituirlo. Nel giugno del 1919, Nitti tornò al governo come presidente del Consiglio e i rapporti con Beneduce tornarono a farsi frequenti $\mathrm{i}^{21}$. Nelle elezioni politiche del novembre del 1919 Beneduce fu eletto deputato e abbandonò per incompatibilità gli incarichi all'Ina e all'Onc, ma continuò a frequentare tanto Nitti quanto Stringher anche negli anni successivi.

Nitti fu preso di mira dagli squadristi e costretto, nel 1924, ad emigrare all'estero. Si mantenne forte e continua invece l'ascesa di Stringher, dovuta al suo rapporto personale con Mussolini. Questo consentirà a Beneduce, nell'ombra e lontano dalla politica, di essere sempre meglio introdotto negli ambienti finanziari italiani e internazionali. Nello stesso tempo egli assicurerà la sua opera silenziosa al fascismo nella fase di transizione dalla democrazia al regime totalitario. Cosicché la parentesi di Ministro del lavoro nel ministero Bonomi, dal giugno del 1921 al febbraio del 1922, costituì una sorta di incidente di percorso cui lo statistico casertano saprà presto ovviare. Con un'accorta presa di distanze, nel clima infuocato delle elezioni del 1924, annuncerà, in modo assai ambiguo, il ritiro dalla scena politica. Nello stesso periodo Nitti fu costretto a lasciare l'Italia.

Gli sviluppi successivi della biografia di Beneduce hanno poco in comune col pensiero nittiano. Per convincersene basterebbe pensare alla convivenza e collaborazione tra pubblico e privato, in una presunta competitività che evidenzia, piuttosto, micidiali forme di conflitto di interessi, conciliabili solo in un regime dittatoriale. Appare così, in tutta la sua chiarezza, l'operazione politica messa in atto, sin dalla fine degli anni Quaranta, da Cuccia, La Malfa e Mattioli che, attraverso il recupero nittiano di Beneduce e la forzatura antifascista e prefascista, puntava in realtà ad evitare che l'Iri fosse ridimensionato, come la Confindustria chiedeva ${ }^{22}$.

La scelta di Beneduce di abbandonare la politica fu annunciata con una lettera pubblica a fine gennaio del 1924. A Bonomi scrisse: «Gli animi sono troppo infatuati o esasperati per intendere la voce della ragione $\gg^{23}$; e al suo vecchio compagno socialista Giuseppe Fusco: «Ho voluto considerarmi solo piuttosto che sorretto da istituzioni che male ritenevano il sacrificio di una vita rivolta a

21 Franzinelli, Magnani, Il finanziere di Mussolini, cit., pp. 56-64.

22 N. De Ianni, Tra industria e finanza, in N. De Ianni e P. Varvaro (a cura di), Cesare Merzagora. Il presidente scomodo, Prismi, Napoli 2004, pp. 3-51 e N. De Ianni, P. Varvaro (a cura di), Il presidente scomodo di uno strano Paese. Atti del convegno Cesare Merzagora. Bilancio storiografico, testimonianze, eredità. Napoli 8 ottobre 2004, Università degli studi Federico II, Napoli 2005, pp. 84-89.

23 Beneduce a Bonomi, 26 gennaio 1924, in Franzinelli, Magnani, Beneduce. Il finanziere di Mussolini, cit., p. 127. 
dare a se stessa questa gioia: guardare nel bianco degli occhi ognuno e sentirsi libero» ${ }^{24}$. È evidente l'ambiguità di tali parole che presupponevano, come gli scriveva ad esempio l'avvocato D'Attorre, animatore del comitato elettorale che nel 1919 e 1921 ne aveva sostenuto la candidatura, «il disagio e la vergogna per questo stato di cose» e non certo l'inizio della collaborazione con un governo che di lì a poco si trasformerà in regime contrario ad ogni libertà politica.

Dopo l'assassinio di Matteotti, Beneduce evitò di affrontare il presente politico anche quando sollecitato direttamente. Alla fine del 1924, la morte del fratello Ernesto fu l'occasione per l'ultimo biglietto con Nitti. Questi, espatriato in Francia, valuterà come un tradimento le successive scelte politiche di Beneduce, contrapponendole a quelle ben più dignitose dell'altro suo vecchio collaboratore Giuffrida.

A Beneduce, grazie all'interessamento di Stringher, era stata affidata sin dal maggio del 1924 la presidenza dell'Istituto di credito di pubblica utilità (Icipu) che si affiancò a quella del Credito per le opere pubbliche (Crediop). I due enti dovranno provvedere, con l'emissione di obbligazioni garantite dallo Stato, al finanziamento di fondamentali settori dell'industria italiana.

Altro tassello fondamentale nell'ascesa di Beneduce fu la nomina nell'ottobre del 1926 alla presidenza della Società italiana per le strade ferrate meridionali (Bastogi) in cui fece da arbitro fra i maggiori azionisti di Comit, Credit ed Edison.

Beneduce non aveva ancora un rapporto diretto con Mussolini ${ }^{25}$. Come Jung, Beneduce collaborò col ministro Volpi ma mentre la collaborazione del primo è offerta al ministro direttamente da Mussolini, quella del secondo avvenne in virtù della mediazione di Stringher. Per tale motivo la sua adesione alla rivalutazione della moneta fu più sfumata e diventerà convinta solo nella seconda metà del $1927^{26}$.

Il ruolo di Beneduce e il suo rapporto personale con Mussolini si accentuò negli ultimi mesi del 1927 quando fu chiaro che americani e inglesi chiedevano, in ottemperanza ai principi che reggevano la comunità delle banche centrali, un maggior potere decisionale di Stringher che era stato messo ai margini da Volpi. Affiancare Beneduce a Stringher costituirà una garanzia tanto per la Banca d'Italia che per il capo del governo. Nel lungo periodo (dal 1928 al 1930) di gestazione della Banca dei regolamenti internazionali, Beneduce sarà l'elemento di continuità da Stringher a Azzolini e vero autorevole rappresentante italiano.

La crisi della sterlina nell'estate del 1931 trovò impreparata l'Italia e mise in forte imbarazzo Beneduce che a fine luglio aveva dichiarato «in qualunque

24 Beneduce a Fusco, 28 febbraio 1924, ivi, p. 128.

25 La corrispondenza personale con i suoi famigliari (18 unità dal 1919 al 1939 in Archivio storico Banca d'Italia, relazione al Fondo n.1 Beneduce a cura di A. Battilocchi) registra la soddisfazione di Beneduce dopo ogni passo che consolida il suo successo personale nell'Italia fascista di Mussolini.

26 Appunto di Beneduce (?), Analisi della situazione economica e finanziaria dell'Italia. Opportunità di arrivare a una fissazione del cambio della lira, in F. Cotula, L. Spaventa, La politica monetaria tra le due guerre, 1919-1935, Laterza, Roma-Bari 1993, pp. 632-634. 
circostanza l'Inghilterra riuscirà a mantenere la stabilità della sua valuta su basi auree $\gg^{27}$ e restò convinto, sbagliando, che l'Inghilterra non avrebbe rinunciato al suo dominio finanziario attraverso la moneta, tanto da dirsi sicuro che presto la sterlina sarebbe rientrata nel gold standard.

L'imbarazzo di Beneduce era dovuto anche alla necessità di dover fronteggiare, insieme ad Azzolini, la rigidità del Ministro delle finanze Mosconi per quanto riguarda la chiusura del bilancio 1931 della Banca d'Italia. Quella che per cinque o sei esercizi era apparsa come una mossa intelligente, ovvero mantenere come riserva aurea le sterline anziché l'oro, con il vantaggio aggiuntivo di una remunerazione su titoli in sterline, diventava ora un buco che si sarebbe potuto evitare soltanto se quelle sterline fossero state per tempo cambiate in oro.

C'è da chiedersi se la scelta, alla fine del 1931, di non seguire la sterlina nella svalutazione rispondesse anche alla necessità di sistemare il bilancio della Banca d'Italia scorporando la perdita derivante dalle sterline e attribuendola al sacrificio cui si era andati incontro rifiutando di svalutare la moneta ${ }^{28}$.

A questo punto resta soltanto da affrontare la questione del ruolo assunto da Beneduce nell'attacco al Credit e alla Banca commerciale di Toeplitz.

La necessità di procedere allo smobilizzo dell'attivo delle ex banche miste, il che equivaleva allo smobilizzo dello stesso Istituto centrale per ingenti somme e le differenze sempre più elevate fra i valori di carico e i valori di borsa delle partecipazioni, determinarono un percorso obbligato e lungo.

Non è da escludere che quello che in sede storiografica è stato descritto come un disorientamento imputabile alla gravità della crisi, all'epoca dello svolgimento dei fatti fosse vissuto dai pochi protagonisti semplicemente come una lunga battaglia da vincere.

In essa era possibile aggiungere a quello economico finanziario dello smobilizzo anche l'obiettivo politico di sconfiggere l'alta banca che, soprattutto dopo l'inizio della politica di rivalutazione della lira, aveva costantemente operato in direzione contraria alla linea di governo.

Va detto che l'alta banca non poteva fare diversamente dopo il 1926 di fronte ad uno scenario rapidamente mutato, con una borsa in caduta, con le industrie in difficoltà per la riduzione della redditività e l'aumento degli oneri finanziari e con una moneta forte ${ }^{29}$. Il portafoglio titoli della Banca commerciale italiana registrò una minusvalenza di circa 2 miliardi di lire dal 1928 al 1931 e di un altro miliardo dal 1931 al 1933 come diretta conseguenza delle scelte valutarie ${ }^{30}$.

27 Lettera di Nathan ad Azzolini, 26 luglio 1931, in Franzinelli, Magnani, Beneduce. Il finanziere di Mussolini, cit., p. 175.

28 De Ianni, Guido Jung e l'intervento dello Stato nell'economia, cit., p. 92. Di estremo interesse è una lettera di Jung ad Azzolini, nell'ottobre 1931, ritrovata fra le carte di Beneduce, in cui, in qualità di presidente dell'Ine, chiede direttive a nome degli esportatori, pena la solidità della lira. Archivio storico Banca d'Italia, Fondo Beneduce, 274, f. 22.

29 Ivi, p. 93.

30 De Ianni, Guido Jung el l'intervento dello Stato nell'economia, cit., p. 107. 
Con questi dati, indipendentemente dalla qualità dei titoli acquistati, difficilmente si sarebbe potuto evitare il fallimento di una qualsiasi società proprietaria di valori azionari. Né era possibile, in quegli anni, per le ex banche miste, chiudere $i$ conti in utile.

Toeplitz esercitò a lungo il suo potere in vario modo ${ }^{31}$. Vendendo lire sul mercato internazionale, costringendo la banca centrale ad allargare l'esposizione del conto di tesoreria o anche mediando, ad esempio con il sottosegretario agli Interni Arpinati che sapeva schierato su posizioni non stataliste ${ }^{32}$.

È singolare che Toeplitz trattasse Beneduce come un suo fiduciario, non avendo capito che invece era da tempo un accanito avversario. Non si spiegherebbe altrimenti che ancora nell'agosto del 1931 Beneduce accettò su richiesta di Toeplitz di farsi promotore di un riservatissimo incontro che si svolse a Firenze con il governatore della Banca d'Italia, Azzolini. L'obiettivo dichiarato era quello di convincere Mussolini e Mosconi ad approvare una mega operazione di doppio riporto con enti statali possessori di titoli di Stato per consentire alla banca di costituire all'estero le garanzie necessarie a far fronte ad una rilevantissima operazione in valuta quantificata in 5 milioni di sterline e 4 milioni di dollari (in totale circa mezzo miliardo di lire) ${ }^{33}$. In un'altra lettera del 22 agosto 1931, Toeplitz confidava molto in un altro incontro combinato da Beneduce presumibilmente con Mussolini ${ }^{34}$.

Si giunse così al salvataggio del settembre-ottobre 1931 quando Beneduce si scagliò senza più nessuna ambiguità contro Toeplitz. Di tale comportamento sono rimaste tracce nei documenti e nella memorialistica. Tutte le ricostruzioni si soffermano sulle urla di Beneduce contro Toeplitz, nell'ottobre 1931, lascian-

31 «La politica di "quota novanta" tenacemente perseguita da Mussolini per oltre un decennio indebolì progressivamente la struttura dell'industria e tolse ogni spazio di azione all'alta banca. Su questo punto la ricerca di Confalonieri è molto utile e portatrice di elementi innovativi. Innanzitutto essa conferma, in maniera molto chiara, la presenza di uno strettissimo nesso tra l'azione del governo fascista e la politica di bilancio degli istituti di credito. In particolare, la rivalutazione, deprimendo i corsi di borsa, impediva alle banche di ridurre l'importo delle partecipazioni e costituì, anzi, la causa principale del progressivo aumento di esso nel bilancio delle banche». N. De Ianni, Banca e mercato nell'opera di Antonio Confalonieri, «Studi storici», 42 (1), 2001, p. 103 e A. Confalonieri, Banche miste e grande industria in Italia, 1914-1933, vol. I. Introduzione; l'esperienza della Banca commerciale italiana e del Credito italiano, Banca commerciale italiana, Milano 1994, pp. 138-195.

32 È noto che Arpinati, nella qualità di sottosegretario agli Interni (ministro era Mussolini) fino all'aprile 1933, curò i rapporti con la sede romana della Banca commerciale e, data la sua nota posizione antistatalista, ebbe contatti con Toeplitz nei delicatissimi mesi in cui si svolse la vicenda. Purtroppo la documentazione non è stata ritrovata. Essa getterebbe una luce sulle cause dell'improvvisa caduta in disgrazia di Arpinati che non può, ragionevolmente, ridursi al litigio col segretario del Partito fascista, Starace.

33 De Ianni, Guido Jung e l'intervento dello Stato nell'economia, cit., p. 90.

34 «Vivamente - scriveva Toeplitz - la prego di trovare il modo - anche tirandolo per i capelli di entrare nel noto argomento. Nessuno meglio di Lei potrà far risaltare l'importanza per il Paese, non solo del superamento delle difficoltà, ma del modo come esse verranno superate, preparando un radioso avvenire», ibidem. 
do intendere che da quel momento la partita si chiuse ${ }^{35}$; la battaglia fu, invece, ancora lunga e si concluse, come si doveva concludere, dopo un altro anno e mezzo di lotte accese ${ }^{36}$.

\section{La nascita dell'Iri}

La temuta e riconosciuta irriducibilità di Toeplitz doveva scontrarsi contro l'insensibilità di Mayer, la ormai dichiarata ostilità di Beneduce, la fredda indisponibilità di Azzolini, la cortese ma pungente critica sulle partecipazioni Sofindit di Jung ed i discorsi pubblici di Mussolini, il quale non perdeva occasione per lanciare messaggi contro i 'cagliostro' dell'economia ${ }^{37}$.

L'inizio del 1932 faceva registrare una situazione sempre più preoccupante della congiuntura, con aziende e banche alle prese con la chiusura di difficili bilanci. Toeplitz chiese fra il maggio e giugno del 1932 all'Imi 600 milioni per la Sip, 400 per l'Italgas, e 300 per la Terni, e di fronte al rifiuto si presentò nuovamente dal duce con una proposta globale di soluzione facendo leva sulle promesse non mantenute della convenzione del 31 ottobre.

Toeplitz chiedeva una emissione di obbligazioni garantite dallo Stato da parte dell'Imi a favore della Sofindit, che li avrebbe girati alla Comit e questa alla Banca d'Italia per liberare la banca dagli oneri sui debiti con l'istituto di emissione, oneri che eccedevano ormai i redditi percepiti. La risposta fu ancora una volta negativa. L'indisponibilità era legata all'intenzione ormai chiara di non far

35 Recentemente, passando in rassegna gli studi di Antonio Confalonieri su Comit e Credit, è parso di poter confermare l'ipotesi «di una voluta inefficacia dell'Imi come strumento per vincere la resistenza di Toeplitz verso una soluzione privatistica della crisi. In altri termini Mussolini, Jung e Beneduce usarono l'Iri per procedere ad una profonda trasformazione dei rapporti di forza fra banca privata e banca pubblica, tra industria privata e industria pubblica in una logica funzionale allo statalismo fascista. Di tali conclusioni l'analisi di Confalonieri sul periodo costituisce l'esatto presupposto confermando come la corretta interpretazione dei documenti degli archivi Comit e Credit resiste anche alla prova di una documentazione originale, diversa e diretta». N. De Ianni, Antonio Confalonieri. "Uno storico di complemento?", relazione al convegno Antonio Confalonieri. Studioso, banchiere, gentiluomo. Nel ventesimo anniversario della scomparsa, Milano, Università Cattolica, 17 marzo 2015, atti a cura di F. Cesarini, A.M. Locatelli e F. Pino, Bancaria editrice, Roma 2017, p. 35. Francesco Cesarini fa risalire allo studio di Confalonieri del 1961 sulle due banche popolari mantovane la passione per gli studi di storia bancaria che portarono Confalonieri a definirsi «uno storico di complemento». Cesarini, Antonio Confalonieri docente e studioso di banca, cit., p. 27.

36 Ne fa fede l'importante lunga lettera di Toeplitz a Jung del 6 ottobre 1932 nella quale l'amministratore delegato della Comit riassunse quanto non fu fatto e si poteva invece fare per il salvataggio della banca in Guarino, Toniolo (a cura di), La Banca d'Italia e il sistema bancario, 1919-1936, cit., pp. 804-823.

37 Nel discorso di Napoli il 25 ottobre del 1931 così si espresse il capo del governo: «Se ci fossero dei diaframmi che volessero interrompere questa comunione diretta del regime col popolo, diaframmi di interessi, di gruppi, di singoli, noi, nel supremo interesse della Nazione, li spezzeremmo!». Franzinelli, Magnani, Beneduce. Il finanziere di Mussolini, cit., p. 196. 
uscire neppure un centesimo senza che Toeplitz rinunziasse alla proprietà della banca e delle partecipazioni. Una sorta di esproprio senza indennizzo.

Il 20 luglio Mussolini operò il cambio della guardia al Ministero delle finanze: Jung al posto di Mosconi. Numerosi i problemi da affrontare. In tema di bilancio dello Stato, contenere il deficit in un contesto di diminuzione delle entrate ed aumento delle uscite. Dal punto di vista finanziario, il consolidamento del debito pubblico che rappresentava il rimedio più proficuo per contenere la spesa attraverso la riduzione degli interessi. Sul piano interno, il problema della perdita provocata dalla svalutazione della sterlina e dell'insufficiente conseguente livello raggiunto dalle riserve auree. Sul versante internazionale l'obbiettivo era l'adesione al blocco dell'oro. Infine, occorreva portare a termine la riforma del sistema bancario e provvedere alla sistemazione dei comparti industriali collegati alla banca mista ${ }^{38}$.

Il nuovo ruolo assunto da Jung gli restituì centralità e maggior potere. Cambiò l'approccio di Beneduce, molto più ossequioso, e sempre più capace di convincere il ministro della bontà delle sue posizioni.

Il capo del governo e il suo Ministro delle finanze dovevano intervenire in una situazione in rapido deterioramento. Fu chiamato alla responsabilità del ministero proprio l'uomo che dall'osservatorio privilegiato della Sofindit aveva potuto rendersi conto dell'importanza della Banca commerciale nell'industria italiana e anche riscontrare la carica eversiva usata da Toeplitz contro la politica monetaria del governo ${ }^{39}$. In sintesi, il pericolo di un potere forte schierato in posizione antagonista e la grande opportunità di consegnare al regime pezzi importanti del sistema industriale.

Con la nomina Jung ottenne da Mussolini l'autorizzazione di continuare ad occuparsi da vicino della Sofindit, attraverso la scelta come presidente del suo fiduciario e parente triestino, Camillo $\mathrm{Ara}^{40}$. Non potendo recarsi a Milano sarà Ara a recarsi $\mathrm{a} \mathrm{Roma}^{41}$.

Dopo alcune settimane di intensa preparazione il progetto di costituzione dell'Istituto per la ricostruzione industriale (Iri) giunse a realizzazione nei primi giorni del 1933. Jung aggirò abilmente l'idea di Mussolini, non condivisa da Beneduce, di costituire un unico ente provvedendo alla incorporazione degli

38 De Ianni, Il ministro soldato, cit., pp. 269-271.

39 G. Toniolo (a cura di), Industria e banca nella grande crisi. 1929-1934, Etas, Milano 1978, p. 286.

40 Jung provvide a sistemare uomini di sua fiducia in posti chiave. Oltre ad Ara alla presidenza Sofindit, Sinigaglia (triestino) all'Ilva (dicembre 1932), Mormino (siciliano) nel Cda Sofindit, e promosse fra i consulenti Reiss Romoli (triestino). Soprattutto fece nominare il fedelissimo Dall'Oro (direttore della filiale di Palermo del Credit) alla direzione generale del Banco di Sicilia. Infine raccomandò per l'assunzione in Banca d'Italia il brillante Enrico Cuccia, figlio del suo amico Beniamino (ottobre 1932).

41 Nel periodo 26 settembre 1932-15 dicembre 1933 Ara ebbe ventinove incontri a Roma con Jung, con frequenza bimensile. Ogni incontro produce una cartellina gialla che Ara portava con sé a Roma in visione al ministro. Archivio storico Intesa Sanpaolo, Banca commerciale italiana, Fondo Sofindit, cart. 395, fasc. 2, sf. Dante Almansi. 
altri esistenti ${ }^{42}$ convincendo il duce che l'uomo giusto fosse Beneduce, anziché Zuccoli ${ }^{43}$.

Le due sezioni, quella di finanziamento e quella di smobilizzo, furono concepite come complementari e parte di uno strumento provvisorio che promuovesse l'assestamento industriale del Paese. Dal punto di vista finanziario, gli organismi (bancari) che avevano avuto bisogno di aiuto non avrebbero più pesato sulla circolazione fiduciaria. Da quello patrimoniale, il sacrificio dello Stato era rilevante perché prevedeva ben venti annualità di 85 milioni cui si aggiungeva il capitale sociale di 100 milioni.

La stampa di regime diede ampio risalto alla nascita dell'Iri anche per la necessità di cominciare ad utilizzare da subito il mercato obbligazionario. La prima emissione di un miliardo fu lanciata dopo una settimana al tasso del 4,5\%, al prezzo di 91 e con la garanzia dello Stato ${ }^{44}$. La Banca d'Italia assunse il coordinamento di un sindacato di collocamento di una quindicina di banche che ne assunsero l'onere.

Beneduce inaugurò in questa occasione un sistema molto prossimo alla doppia intermediazione poiché gli istituti erano 'costretti' a sottoscrivere le quote e a collocarlo a proprio rischio tra la clientela. I risultati definitivi del collocamento furono salutati sulla stampa con toni trionfalistici.

\section{La missione negli Stati Uniti e gli interventi alla conferenza economica di Londra}

L’anno 1932 fu assai duro per le imprese italiane alle prese con una crisi economica acuita dalle scelte monetarie del regime. A marzo del 1933, Toeplitz svolse la sua ultima relazione all'assemblea Comit, prima dell'abbandono di ogni carica operativa e della nomina a vicepresidente. Uscì sconfitto dopo una lunga battaglia e con la frustrazione di vedere molti dei suoi uomini allineati col nuovo corso.

In aprile, Mussolini ricevette un invito ufficiale dal neoeletto presidente degli Stati Uniti Roosevelt di recarsi in America per intese in vista della conferenza economica di Londra, programmata per metà giugno del 1933. Il presidente, in attesa di avviare il suo New Deal, dichiarò improvvisamente il 20 aprile l'inconvertibilità del dollaro, con conseguente svalutazione della mone-

42 Beneduce sostenne che quelle fusioni erano inattuabili, pena lo smantellamento di emissioni obbligazionarie collocate verso una platea di risparmiatori. Dato il suo collocamento nel regime ancora precario, questa posizione tradiva probabilmente il timore di veder scomparire gli enti da lui diretti. Ed infatti, come nota opportunamente Giulianelli, sette anni dopo, nel 1940, Beneduce «non solo accetta di buon grado la sottomissione dei suoi enti all'Imi, ma se ne arroga addirittura la paternità»; R. Giulianelli, Armatori, banche e Stato. Il credito navale in Italia dall'Unità alla prima crisi petrolifera, il Mulino, Bologna 2017, p. 184.

43 Jung incontrò Beneduce e non Zuccoli; aveva in animo di proporre il suo nome a Mussolini e si mosse con circospezione per evitare di bruciarne la candidatura. Non sembri strano l'accostamento di Zuccoli a Beneduce. Zuccoli era molto stimato ed anche molto temuto per le sue relazioni nel Partito fascista. De Ianni, Il ministro soldato, cit., pp. 282-285.

${ }^{44}$ L'emissione di un miliardo dell'Iri, «Corriere della sera», 1/2/1933. 
ta, mentre si disse risolutamente contrario a qualsiasi ipotesi di cancellazione dei debiti di guerra.

Mussolini decise di inviare negli Usa, quale suo delegato, il Ministro delle finanze che partì il 26 aprile ${ }^{45}$. Durante il viaggio Jung dovette fronteggiare e scoraggiare, d'intesa con la Banca d'Italia e il governatore Azzolini, l'azione di alcune primarie imprese italiane come la Fiat intenzionate ad estinguere i propri debiti in dollari approfittando della svalutazione. Nel caso specifico si sarebbe trattato di 110 milioni di dollari da acquistare proprio mentre il governo si apprestava a trattare con gli americani per l'annullamento della rata di 14 milioni scadente a giugno, onorata con la cifra simbolica di un milione ${ }^{46}$.

La stampa americana diede ampio risalto alla missione di Jung e ai tre incontri con Roosevelt. Sulla questione dei debiti di guerra il delegato italiano, sin da dieci anni prima, era stato tra i pochi a riconoscere il diritto americano alla non cancellazione del debito. Il problema più delicato era certamente quello dell'ortodossia monetaria, reso ancor più attuale dall'abbandono della parità aurea del dollaro, come nel 1931 aveva fatto la sterlina.

Incombeva intanto la conferenza economica di Londra, i cui lavori si aprirono il 12 giugno. Dopo tre settimane di febbrili trattative, in cui l'Italia fu fra i paesi che più ostinatamente si batterono per la fissazione di nuove parità, il 3 luglio Roosevelt annunciò l'indisponibilità americana. Fu un brusco richiamo alla realtà. I lavori della conferenza furono influenzati dalla chiusura americana e molto diverso fu lo spirito degli interventi fra prima e dopo.

Il primo italiano ad intervenire fu naturalmente il capo della delegazione Jung che insistette sull'opportunità di tre principali obbiettivi: aumento del livello dei prezzi; stabilizzazione delle monete; eliminazione degli ostacoli agli scambi commerciali. Si disse convinto che la crisi economica non potesse essere affrontata con iniezioni, definite forzate, di credito bensì soltanto col governo del risparmio, considerato il fattore dominante della possibile ripresa ${ }^{47}$. È appena il caso di osservare come la posizione italiana considerasse le necessarie iniezioni di liquidità come turbative e non sostegno del mercato. Alla luce di quanto è accaduto nei decenni successivi, in termini di politica economica e ruolo delle banche centrali, si trattava dell'enunciazione di una cultura economica arretrata e inadeguata a fronteggiare la crisi economica mondiale.

I due interventi di Beneduce alla conferenza rappresentano la cifra tecnica della posizione italiana.

Il primo, il 21 giugno, ebbe per oggetto la stabilizzazione e la base aurea. Richiamandosi a quanto sostenuto da Jung e polemizzando con il cancelliere dello scacchiere britannico Chamberlain, Beneduce disse che i delegati italiani pensavano, per riequilibrio di costi di produzione e prezzi, ad una soluzione

45 F. Suvich, Memorie. 1932-1936, a cura di G. Bianchi, Rizzoli, Milano 1984, p. 192.

46 Archivio storico Banca d'Italia, Direttorio Azzolini, corda 26, fasc. 1, ff. 66-67, 29-30/4/1933 e Fondo Beneduce, corda 309, ff. 2231 e sgg., 22/4-2/5 1933.

47 De Ianni, Il ministro soldato, cit., p. 295. 
economica, richiamando il pericolo di un intervento finanziario o di un'azione combinata nel campo finanziario ed economico.

Il secondo lo tenne il 4 luglio, il giorno dopo la rottura con Roosevelt. Beneduce, lasciata da parte ogni prudenza, si lanciò in uno scomposto attacco alla politica economica americana in generale e al partito democratico in particolare ${ }^{48}$. Affermò che la politica americana dell'ultimo decennio era stata caratterizzata da aggressività nei confronti degli altri paesi e soprattutto nei confronti del commercio europeo. Le linee di azione di questa aggressione erano state la politica di espansione del credito da una parte e la critica del protezionismo dall'altra, in modo da creare una sorta di dumping a vantaggio dell'economia americana. Nella recente competizione elettorale, si erano, secondo Beneduce, fronteggiati il partito conservatore, difensore della prosperità e quello democratico che intendeva sperimentare l'azione monetaria come strumento di liquidazione della crisi economica. Con la vittoria di Roosevelt gli americani avevano scelto l'illusione di superare la crisi senza sforzi e senza perdite reali. L'Europa con la sua esperienza di secoli di civiltà latina aveva scelto, invece, un'altra strada, quella di una valuta ancorata all'oro, quale presidio di contenimento economico.

Naturalmente quello che Beneduce, Jung e Mussolini presentavano come un conflitto tra l'ordine e il disordine era in realtà una sfida tra il vecchio e il nuovo, come sottolineò opportunamente Keynes in un articolo di sostegno a Roosevelt. «È sostanzialmente una sfida lanciata a noi perché decidiamo se battere le vecchie strade infauste o esplorarne di nuove, nuove per statisti e banchieri, ma non nuove al pensiero, poiché conducono alla moneta regolata dell'avvenire il cui esame è stato l'argomento centrale dell'economia postbellica» ${ }^{49}$.

\section{La sistemazione bancaria e le convenzioni del marzo 1934}

La sistemazione delle ex banche miste, ivi compresa la loro nazionalizzazione, si realizzò attraverso specifiche convenzioni nel marzo del 1934. Le tre banche giunsero all'appuntamento in condizioni diverse, ma il risultato, frutto di una razionalizzazione decisa a monte, fu uguale per tutte e tre.

Due i documenti da analizzare in proposito: quello elaborato dal direttore generale dell'Iri Menichella e inviato dall'Iri al capo del governo il 5 dicembre 1933 e la ricostruzione delle varie tappe dell'operazione fatta da Beneduce nel marzo del 1935, all'atto della presentazione del bilancio Iri al 31 dicembre $1934^{50}$. Tanto Menichella che Beneduce insistevano nel definire il provvedimento adot-

48 Archivio storico Banca d'Italia, Fondo Beneduce, corda 342, riportato da M. De Cecco (a cura di), L'Italia e il sistema finanziario internazionale, 1919-1936, Laterza, Roma-Bari 1993, pp. 454-458.

49 R.F. Harrod, La vita di J.M. Keynes, Einaudi, Torino 1975, p. 520.

so Il primo documento è in F. Cotula, C.O. Gelsomino, A. Gigliobianco (a cura di), Donato Menichella. Stabilità e sviluppo dell'economia italiana, 1946-1960, vol. I. Documenti e discorsi, Laterza, Roma-Bari 1997, pp. 74-124. Il secondo in Guarino, Toniolo (a cura di), La Banca d'Italia e il sistema bancario, 1919-1936, cit., pp. 850-871. 
tato come «profondamente rivoluzionario» perché con la sua attuazione si era guardato in faccia alla realtà applicando «rimedi totalitari».

Beneduce chiariva che, sebbene venuti a conoscenza del pubblico come una determinazione improvvisa del governo, in realtà essi erano stati meditati da lungo tempo essendo nel grande riserbo cominciato lo studio della situazione bancaria sin dall'autunno del 1933. Tutta la responsabilità del deterioramento della situazione veniva attribuita esclusivamente al management delle banche piuttosto che alla politica di rivalutazione della moneta che dal 1926 al 1932 aveva portato il cambio della sterlina da 150 a 60 .

È poco credibile la data dell'autunno 1933 come quella di inizio degli studi. Fra le carte di Jung vi è un documento datato 4 marzo 1933, frutto di un lavoro di équipe tra vecchi e nuovi dirigenti delle banche, poi rivisto da Jung, con il quale si chiedeva al capo del governo di portare sia la Società finanziaria italiana (Sfi) che la Società finanziaria industriale italiana (Sofindit) sotto il controllo dello Stato attraverso l'Iri ${ }^{51}$. Tale promemoria è molto indicativo dello spirito con il quale venne combattuta la battaglia per la conquista delle ex banche miste.

La stessa data del 4 marzo è assai indicativa perché precede di pochi giorni l'assemblea in cui viene estromesso Toeplitz dalla direzione della Comit, mentre segue di poche settimane il trionfale primo collocamento obbligazionario dell'Iri.

Il documento inizia con un chiarimento circa la convenzione del 31 ottobre 1931, studiata per permettere il controllo di Sofindit e quindi, a catena, di Comofin e Comit, il che rivela come, sin da allora, il regime puntasse direttamente alla banca milanese. Si specifica inoltre come abbia potuto esercitare tale controllo sin dal $1^{\circ}$ gennaio 1932 (si noti, data di insediamento di Jung alla presidenza Sofindit) non con «un effettivo controllo della gestione che non sarebbe praticamente realizzabile $[\ldots] \mathrm{ma}$ attuando la sua volontà in materia di indirizzo». Si spiega ancora l'impossibilità di soddisfare le continue richieste di aiuti finanziari che provengono dai dirigenti perché, a causa del sistema a catena, tali aiuti sarebbero andati a vantaggio dei vecchi azionisti.

Il promemoria si sofferma anche sulla situazione azionaria del Credito italiano e sul fatto che non esista più né il capitale sociale delle due controllanti Sfi e Elettrofinanziaria, per via delle perdite sul portafoglio titoli, né quello dello stesso Credito italiano assorbito dalle perdite dei suoi crediti verso le controllanti. Inoltre, vi si legge che nell'estate del 1932, pochi giorni dopo la nomina a ministro di Jung, ci fu una richiesta del Credito italiano di un ulteriore contributo di 990 milioni dopo i 330 concessi con la convenzione del febbraio del 1931 e di una sistemazione dei pacchetti azionari a cascata in modo che alla fine agli amici del Credito italiano (fra cui i principali azionisti erano Feltrinelli e Pirelli) fosse garantito il controllo della banca. Inutile dire che Jung si oppose risolutamente a questa sistemazione perché i capitali di Elettrofinanziaria e Credito italiano, ormai inesistenti, sarebbero stati ricostituiti a spese dello Stato.

51 Archivio Jung Palermo, corda 16, ff. 3-7, 4/3/1933, Risanamento bancario. 
Attraverso il controllo a catena, tale ricostituzione sarebbe andata ad esclusivo vantaggio degli amici del Credito italiano.

Nell'archivio del Credito italiano c'è un interessantissimo carteggio fra il dirigente del Credit Malvezzi e De' Stefani avente per oggetto le preoccupazioni di una fusione con Comit. L'ex ministro consiglierà di affidarsi a Jung dicendosi tranquillo che né lui né tantomeno Mussolini avrebbero operato per unire «una pera bacata con una pera sana ${ }^{52}$.

Il documento di Jung si conclude con l'enunciazione di un principio ispiratore fondato sull'iniziativa privata che suona quasi beffardo date le circostanze. L'intervento dello Stato aveva assicurato un'opera risanatrice nella gestione delle ex banche miste senza tenere in alcun conto i capitali privati e gli assetti azionari preesistenti. Restano infine forti dubbi sul carattere progressista e riformatore di una posizione che tendeva a favorire il risparmio obbligazionario (ma non quello azionario) in un conflitto aperto dallo Stato fascista contro il grande capitale, all'interno di uno schema di rigorosa ortodossia monetaria.

Tale posizione così rigidamente perseguita sarà abbandonata nell'ottobre del 1936 per far strada alla politica di spesa che l'impero imponeva e alla politica autarchica di guerra. Vi sarà allora un drastico ritorno ai valori di fine 1927 (92 lire contro sterlina e 19 contro dollaro) presentato come un semplice riallineamento.

Possiamo chiederci: vi erano degli ostacoli presenti nel 1931 venuti poi a mancare nel 1936? La risposta è nella complessità di un assetto di politica industriale e bancaria che nel 1931 non era stato ancora realizzato, con un settore privato in difficoltà, in un sistema che sfavoriva coloro che esportavano rispetto a coloro che importavano. A partire da allora, il fascismo, grazie alla crisi economica e all'intervento dello Stato nell'economia, avviò un processo di riorganizzazione che nel 1936 appariva ormai pienamente realizzato ${ }^{53}$.

\section{La liquidazione della Sofindit}

Nel corso del 1934 Beneduce cercò di concentrare a Roma la gestione degli affari, attraverso la proposta di fusione della Sofindit nell'Iri, mentre Ara e Di Veroli facevano pressione per mantenere una propria autonomia. Nella discussione s'intromise anche Sinigaglia che oltre ad essere dal dicembre 1932 presidente dell'Ilva, era stato anche nominato da Jung consulente della Sip. In sintesi, Beneduce accarezzava l'idea di dare forza all'Iri mantenendo le partecipazioni profittevoli non retrocesse ai privati. Sinigaglia pensava viceversa che l'Iri non potesse comportarsi come una qualsiasi società privata.

Nella documentazione di archivio vi sono tracce di una forte opposizione alla linea accentratrice di Beneduce man mano che essa cominciò a svilupparsi con la richiesta di lasciare a Milano almeno una parte delle partecipazioni ac-

\footnotetext{
52 Archivio storico Credito italiano, Segreteria Alta direzione pratiche sf. A, N. 18, Colloqui De' Stefani-Malvezzi, 18 e 23 gennaio 1933.

53 De Ianni, Il ministro soldato, cit., pp. 306-307.
} 
quisite. Per il successo dell'iniziativa sarebbe stato naturalmente necessario il consenso del ministro, che non ci fu.

Due lettere fortemente critiche nei confronti di Beneduce testimoniano i passi.

La prima è dell' 8 aprile 1934. Sinigaglia cercò di convincere Jung dell'errore che, secondo lui, insieme a Beneduce stava commettendo e cioè l'accentramento enorme di un alto numero di aziende sotto la direzione di un uomo solo ${ }^{54}$.

La seconda lettera del 20 marzo è di Camillo Ara. La questione riguardava una richiesta avanzata due mesi prima, rimasta senza risposta, relativa alla costituzione di una nuova fiduciaria per evitare il concentramento nell'Iri di tutte le società industriali. Ara non voleva la messa in liquidazione della società da lui presieduta, proponendo che la fiduciaria avrebbe controllato l'attività dei settori tessile, minerario, siderurgico e meccanico, chimico, del trasporto marittimo. All'Iri sarebbe rimasta la responsabilità delle società elettriche, delle immobiliari, dei trasporti terrestri e del settore finanziario, incluse naturalmente le banche. Dichiarò di non voler collaborare con Beneduce per l'eccessiva tendenza alla centralizzazione e per i metodi autoritari da lui usati ${ }^{55}$. Parole dure verso il presidente dell'Iri e non meno severe all'indirizzo di Jung, accusato di essere sottomesso a Beneduce.

Ara, dopo qualche settimana di polemiche, ridimensionò fortemente le sue critiche fino a farle rientrare del tutto. Accettò il piano Beneduce, fu nominato commissario liquidatore della Sofindit e poi vicepresidente, senza cariche operative, dell'Iri.

Jung preferì abbandonare i suoi vecchi amici e, di lì a poco, subirà anche lui la furia vendicatrice di Beneduce ${ }^{56}$.

\section{La conversione della rendita}

Il problema di un crescente deficit di bilancio era particolarmente avvertito e Jung aveva cercato di conseguire miglioramenti con una politica di contenimento e sacrifici. Abbiamo già visto come il tema della conversione della rendita rientrasse fra gli obiettivi di governo ma è chiaro che bisognava muoversi su questo tema con grande attenzione. Alla fine del 1932 il debito pubblico ammontava a circa 96 miliardi con un costo degli interessi intorno ai 5 miliardi.

54 Archivio Jung Palermo, corda 29, fasc. 5, sf. 1, Sinigaglia a Jung, 8 /4/1934.

55 «Con la società fiduciaria - scriveva Ara - non mi troverei, naturalmente davanti ai fatti compiuti, agirei con il mio metodo; non prevarrebbero i criteri di altri, salvo eccezioni e sempre salvo esame e discussione. Penso che l'autonomia delle teste di Iri in Milano sarebbe presto ferita. Possibili conflitti. Possibile rottura, spiacevolissima. Convegno con $\mathrm{B}$ [eneduce] per domani mercoledì. L'esposizione e le decisioni saranno del ministro?». Ivi, corda 14 , fasc. 4 , f. 11.

56 È singolare osservare come di tutto questo non ci sia traccia nei recenti volumi sulla Storia dell'Iri. In particolare V. Castronovo (a cura di), Storia dell'Iri, vol. I. Dalle origini al dopoguerra. 1933-1948, Laterza, Roma-Bari 2012. 
Nel corso del 1933 Jung inaugurò una politica di ribasso del costo del denaro, facendo leva sul tasso ufficiale di sconto. Dal $5 \%$ fu portato al $4 \%$ nel gennaio del 1933, al 3,5\% nel settembre e al 3\% nel mese di dicembre.

Il 10 gennaio 1934 vi fu un'offerta di buoni del Tesoro novennali al $4 \%$ al prezzo di 99 , sottoscritta per oltre 7 miliardi contro i 4 offerti. L'operazione serviva a preparare la conversione del consolidato, già decisa, che fu effettivamente lanciata il 3 febbraio. La proposta prevedeva la conversione di quattro prestiti consolidati al 5\% con un prestito redimibile al 3,5\%. Il risparmio previsto sugli interessi del debito era di circa un miliardo. L'operazione riuscì perfettamente al momento (fu infatti convertito il $99,8 \%$ del totale), salvo presentare il conto l'anno dopo ${ }^{57}$. Dalle carte di Beneduce si ha la conferma di una collaborazione intensa con Jung anche per le difficoltà tecniche di una conversione di prestiti da irredimibili (perpetuo) a redimibili (scadenza lunga) ${ }^{58}$.

La situazione economica generale, a partire dalla fine della prima metà del 1934 cominciò sensibilmente a peggiorare. Jung aveva impostato la sua politica con assoluta rigidità e l'aveva trasformata in una questione di principio.

A maggio la lira fu sottoposta ad un massiccio attacco speculativo al ribasso. Mussolini si chiese e chiese al suo ministro di esaminare la convenienza di una svalutazione della lira. Jung rispose con uno studio di venti pagine a difesa del suo operato. Le cause della debolezza della lira erano dovute al drastico calo delle esportazioni e un disancoraggio dalla attuale parità aurea sarebbe stato equivalente all'abbandono del tallone aureo. Jung pensava ai risparmiatori italiani che avevano accettato la conversione al 3,5\% dal $5 \%$ con la motivazione che essa era dovuta alla rivalutazione della lira. Svalutare avrebbe significato tradirli, per cui pensava piuttosto di inasprire le misure per colpire con la più grande energia la fuga dei capitali all'estero ${ }^{59}$.

\section{L'emorragia di oro e il cambio della guardia: gennaio 1935}

La situazione dei cambi internazionali dal 1934, da quando cioè gli Stati Uniti decisero di riportare il dollaro verso la convertibilità, si fece molto volatile. In particolare, il livello fissato pari al $59 \%$ della vecchia parità avvicinava il cambio con la sterlina al rapporto di 5 contro 1 , ben diverso da quello 4 contro 1 della fallita trattativa del giugno/luglio precedente alla conferenza di Londra. Per

57 Archivio storico Banca d'Italia, Fondo Beneduce, corda 306, fasc. 3, ff. 80 e sgg., Promemoria per S. E. Beneduce.

58 Tra le carte di De' Stefani c'è la registrazione di una telefonata col ministro alle ore 8.30 del 3 febbraio, giorno di partenza dell'operazione. L'ex ministro lamentava di non essere stato consultato. Uno Jung assai imbarazzato rispose di aver fatto tutto da solo. De' Stefani replicò che lo studio della conversione, da informazioni assunte, non proveniva dagli uffici del ministro, alludendo evidentemente alla collaborazione di Beneduce. Ivi, Archivio De' Stefani, corda 38, fasc. 1/33, Conversazione telefonica De' Stefani-Jung, 3/2/1934.

59 Archivio Jung Palermo, corda 22, fasc. 6, ff. 2-23, Esame della convenienza della svalutazione della lira, Jung al duce, 16/5/1934. 
la lira ciò equivalse ad un'ulteriore stretta con un cambio che per tutto il 1934 oscillò tra 58 e 59 contro la sterlina e 11,7/12 contro il dollaro.

Gli effetti sulla bilancia commerciale furono evidenti. Tra il 1933 e 1934, mentre le importazioni aumentarono da 7,35 a 7,58 miliardi, le esportazioni diminuirono da 5,75 a 4,96 miliardi con un deficit commerciale che passò quindi da 1,6 a 2,6 miliardi $^{60}$.

Ma il punto nevralgico per Mussolini era certamente il livello delle riserve auree. Il 31 dicembre 1931 esse erano scese a 7,797 dai 12,1 miliardi del dicembre 1927 e continuarono a scendere nella prima metà del 1932. Con l'avvento di Jung al governo si registrò un sensibile miglioramento che non impedì però una ulteriore diminuzione a fine $1932(7,143)$ e solo un piccolo miglioramento a fine $1933(7,3997)$. Con il 1934 la caduta riprese con intensità portando il saldo a 6,5 il 30 giugno 1934 e a 5,88 il 31 dicembre. Nonostante ogni sforzo, i dati deprimenti mostravano in misura evidente che la parità aurea era fissata a un valore che il mercato considerava eccessivo.

Già dall' 8 dicembre 1934 fu reintrodotto il monopolio sul commercio delle valute affidato all'Istituto italiano cambi con l'estero, mentre fu sospesa la convertibilità della lira. Tale politica di restrizioni si affiancava ad una politica industriale sempre più centrata sui consorzi obbligatori. La congiuntura incoraggiava un intervento dello Stato crescente ma con effetti negativi di burocratizzazione degli apparati. In una lettera del 25 giugno 1934 Mussolini si era rivolto con toni inequivocabilmente ultimativi contro il suo ministro considerando inaccettabile la situazione e minacciando provvedimenti se si fosse scesi sotto i 6 miliardi ${ }^{61}$.

Fu questo molto probabilmente il motivo del cambio della guardia che fu comunicato coi soliti modi sbrigativi il 24 gennaio 1935. Mussolini ringraziava Jung per le grandi operazioni di conversione e per il deciso avviamento al pareggio del bilancio ${ }^{62}$. Non una parola invece sulla soluzione del problema bancario e sulla nascita dell'Iri che pure aveva coinvolto in maniera così diretta Jung. Evidentemente Mussolini aveva rimosso o riconosciuto soltanto a Beneduce quei meriti.

Sull'uscita di Jung dal ministero esiste una nota di una informatrice della polizia politica che riferisce di un acceso scontro tra Jung e Beneduce relativo all'operazione di conversione, in cui pare che Beneduce scaricasse su Jung la responsabilità di consigli che lui aveva dato. La questione sarebbe finita davanti al duce con conseguenti dimissioni presentate dal ministro e con estremo fastidio respinte. L'informatrice nota che Mussolini è infastidito dalla mancata osservanza della regola secondo cui le dimissioni può ordinarle soltanto lui ${ }^{63}$.

60 De Ianni, Il ministro soldato, cit., p. 320.

61 R. De Felice, Mussolini il duce, vol. I. Gli anni del consenso, 1929-1936, Einaudi, Torino 1996 (1 ed. 1974), pp. 140-141.

62 De Ianni, Il ministro soldato, cit., pp. 330-331.

63 M. Franzinelli, I tentacoli dell'Ovra. Agenti, collaboratori e vittime della polizia politica fascista, Bollati Boringhieri, Milano 1999, p. 668. 


\section{Beneduce e la resa dei conti con Ara, Sinigaglia, Mazzini, Puricelli, Feltrinelli}

Con l'uscita di scena Jung ruppe ogni rapporto con Mussolini, e Beneduce rimase arbitro incontrastato della situazione, vero dittatore dell'economia italiana nei modi e nei termini che lo avevano reso più che fascista, un mussoliniano ${ }^{64}$. Scrisse con Menichella la riforma bancaria del 1936 con la quale si sancì definitivamente la separazione del credito ordinario da quello a medio e lungo termine. E realizzò nel 1937 la trasformazione dell'Iri in ente permanente, mettendo a disposizione di Mussolini nell'Italia autarchica una macchina da guerra. De' Stefani scrisse che Beneduce era dotato di una grande capacità di persuasione grazie alla quale aveva convinto tutti che nelle scelte finanziarie del Paese non si potesse prescindere da lui ${ }^{65}$. Lo circondava un alone di riservatezza e di mistero.

Per la nomina di Ara a senatore, Jung aveva ottenuto da Mussolini una promessa che non fu mantenuta dopo i forti scontri con Beneduce ${ }^{66}$.

Peggio andò a Sinigaglia, i cui contrasti con Beneduce datavano ancor prima. Coerentemente col carattere impulsivo, Sinigaglia scrisse a Beneduce per lamentare la mancata assegnazione di dividendi agli azionisti Sip per l'acquisizione della azienda telefonica. Beneduce rispose che, in assenza dei provvedimenti del governo fascista, agli azionisti Sip non sarebbe rimasta altra alternativa che il dolersi «delle fauci Panzarasa-Comit ${ }^{67}$. Abbiamo visto come Sinigaglia spingesse la polemica con Beneduce oltre i limiti del buon senso. Sull'attacco alla sua etica, Beneduce tacque, ma non gradì. E quando un anno dopo venne a sapere da Puricelli che c'era un giro di tangenti che riguardava una azienda di proprietà di Sinigaglia, fu inflessibile e chiese ed ottenne le sue dimissioni dalla presidenza dell'Ilva ${ }^{68}$.

Molto diverso il caso dell'industriale e deputato piemontese Giuseppe Mazzini cooptato nel consiglio della Sofindit da Jung probabilmente per compiacere Pirelli e la Confindustria. Mazzini però non era uno yes men. Voleva vederci chiaro in quella interminabile serie di bilanci e sulle responsabilità che competevano agli amministratori. E certamente fastidiosa fu la critica rivolta da Mazzini a Beneduce sulla sistemazione della Sip. L'industriale piemontese si scagliò contro gli alti tassi di interesse nonostante l'obiettiva convenienza a tenerli bassi anche per favorire l'operazione di conversione della rendita. Tassi alti per agevolare il collocamento delle obbligazioni dell'Iri, nonostante la garanzia dello Stato e «una tecnica bancaria che ne garantisce il successo anche all'infuori della fiducia e

64 P. Melograni, L'adesione di Beneduce al regime mussoliniano, in P. Armani et al., Alberto Beneduce e i problemi dell'economia italiana del suo tempo. Atti della giornata di studio per la celebrazione del $50^{\circ}$ anniversario dell'istituzione dell'Iri, Caserta, 11 novembre 1983, Edindustria, Roma 1985, pp. 175-178.

65 De' Stefani, Baraonda bancaria, cit.

66 Una prima ondata della campagna antisemita rese il provvedimento poco opportuno e successivamente impossibile.

67 Archivio storico Intesa Sanpaolo, Sofindit, cart. 405, fasc. 6, sf. 2, Sinigaglia Beneduce, 28/8/1933 e Beneduce a Sinigaglia, 31/8/1933.

68 Archivio Jung Palermo, corda 29, fasc. 5, sf. 1, f. 39 e fasc. 1, sf. 2, ff. 18-20. 
della bontà dell'investimento». Tassi alti che però aumentavano gli oneri per lo Stato, facevano calare i corsi delle emissioni precedenti e riducevano le possibilità di finanziamento per tutte le altre aziende. Tali prestiti avvenivano ad un tasso di circa il 6\% netto; per le altre aziende la raccolta di danaro costava almeno due punti in più. Come si poteva resistere e investire in un momento di crisi così grave? Inoltre, a parere di Mazzini, l'impostazione dell'Iri e degli «anabattisti della Sip», come egli chiamava i collaboratori di Beneduce, era sbagliata anche perché l'intero piano di sistemazione si basava sui ricavi della gestione industriale di un anno scarsamente rappresentativo quale era l'esercizio 1932. Sbagliata anche per la sottovalutazione di impianti che nascondevano un potenziale di elevata redditività. Se si azzerava il valore di quegli impianti i nuovi azionisti si sarebbero trovati un indebito vantaggio ascritto a proprio merito ma pagato dai vecchi azionisti. C'era infine un palese conflitto di interessi fra il trattamento riservato alla Sip e quello ad altre grandi aziende private (Edison e Sade) e Mazzini si chiedeva se questo sarebbe stato possibile in un regime di libera concorrenza ${ }^{69}$.

Ancora più indicativa e atipica la vicenda della Puricelli. L'ex partecipazione della Comit nell'azienda delle strade seguì un percorso diverso da molte altre, riducendosi dal 1929 della metà ad un terzo e restando poi oggetto di contesa fino al 1936. Del tutto particolare era inoltre il rapporto dell'azienda con Sofindit e soprattutto con l'Iri, la cui sezione smobilizzi operò, nel corso dei primi anni Trenta, una serie numerosa di risanamenti con retrocessione a privati. Non è il nostro caso, dato che la società fu sottoposta a una drastica cura a base di continui salassi che puntavano a costringere Puricelli a cedere la sua azienda ed uscire di scena. Va sottolineato anche come, diversamente da molte altre esperienze in cui lo Stato era chiamato a subire notevoli perdite, qui esso anticipa fondi, ma recupera tutto in pochi anni e con adeguati interessi. La vicenda di cui si tratta non rientra, dunque, fra quelle di una mentalità tipica di molti industriali e finanzieri sintetizzabile nell'assunto: «socializzare le perdite e privatizzare i guadagni $\gg^{70}$.

Avvolta ancora nel mistero ma sostanzialmente chiara nelle sue modalità la vicenda di Carlo Feltrinelli. Accusato di detenere larghe disponibilità all'estero intestate a familiari, Feltrinelli comincerà a sentirsi perseguitato da Beneduce. Alla fine del 1935, convinto che lo si volesse disonorare, morirà in circostanze tragiche, probabilmente suicida, nell'anticamera di Beneduce che gli aveva chiesto le dimissioni da tutte le cariche ${ }^{71}$.

69 De Ianni, Guido Jung e lo Stato imprenditore, cit., pp. 100-103. Mazzini era stato eletto alla Camera nel 1921, rieletto nel 1924 nel listone e poi confermato nel 1929 nelle elezioni del plebiscito. Nella primavera del 1934 non fu confermato alla Camera e nel 1935 tornò alla presidenza della Lega industriale di Torino.

70 N. De Ianni, Vecchi e nuovi documenti sullo "Stato industriale". Di un episodio nel conflitto pubblico-privato: $i$ riassetti della Puricelli, in A.M. Falchero et al. (a cura di), La storia e l'economia. Miscellanea di studi in onore di Giorgio Mori, vol. II, Edizioni Lativa, Varese 2003, pp. 291-316.

71 L. Segreto, I Feltrinelli. Storia di una dinastia imprenditoriale (1854-1942), Feltrinelli, Milano 2011. 
10. Epiloghi

Dopo il gennaio del 1935 Jung proseguì la sua vicenda esistenziale verso una sofferta parabola discendente.

Nel 1935 fu tra i primi a partire per l'Africa orientale alla conquista dell'impero, ricevendo la sua quarta medaglia d'argento che gli sarà consegnata dal duce nel corso di una cerimonia pubblica il 31 maggio 1936.

L'attività della ditta che aveva già subito un forte ridimensionamento per la politica del governo che lui stesso era stato chiamato a realizzare, subì gli effetti dei provvedimenti razziali e fu costretta alla chiusura il 30 giugno 1939.

In materia militare Jung resisté a molte prove, ma subì un colpo decisivo quando fu improvvisamente cancellato dai ruoli dell'esercito.

Lo sbarco degli anglo americani nel luglio del 1943 lo colse a Palermo pronto a dare il suo contributo in caso di necessità. Badoglio lo nominò Ministro delle finanze del suo governo.

Terminata questa esperienza fece domanda per essere inviato a combattere i tedeschi con l'esercito italiano.

Dopo la Liberazione ricostituì la ditta con il nipote, ma non volle riprendere l'attività politica nonostante le sollecitazioni ricevute e le persecuzioni razziali subite.

Dopo le elezioni del 18 aprile 1948 valutò con ironia il coraggio del suo amico Sinigaglia di essere passato in cinquant'anni dai nazionalisti a De Gasperi.

Morì a Palermo nel dicembre del 1949.

Nell'estate del 1936 Beneduce fu colpito da un ictus che gli tolse l'uso della parola e gli paralizzò una parte del corpo ${ }^{72}$. Con grande forza d'animo riuscì a riprendersi.

Nell'aprile del 1939 fu nominato senatore nella categoria degli ex ministri a sottolineare il suo periodo prefascista e fu costretto a prendere la tessera del Pnf automatica con la nomina ${ }^{73}$.

Nel novembre del 1939 si dimise dalla presidenza dell'Iri e nell'aprile del 1940 dal Crediop e l'Icipu. Rimase fino alla morte alla presidenza della Bastogi.

Visse gli ultimi anni da fascista e da mussoliniano, coltivando la certezza di aver bene operato per il suo Paese.

Morì a Roma nell'aprile del 1944.

72 Franzinelli, Magnani, Beneduce. Il finanziere di Mussolini, cit., p. 266.

73 F. Bonelli, Alberto Beneduce (1877-1944), in A. Mortara (a cura di), I protagonisti dell'intervento pubblico in Italia, Franco Angeli, Milano 1984, p. 355. 